\begin{tabular}{|c|l|}
\hline Title & Excitation of cavitation bubbles in low-temperature liquid nitrogen \\
\hline Author(s) & Sasaki, Koichi; Harada, Shingo \\
\hline Citation & $\begin{array}{l}\text { Japanese Journal of A pplied Physics (JJA P), 56/6), 68002 } \\
\text { https://doi.org/L0.7567/JJA.56.068002 }\end{array}$ \\
\hline Issue Date & 2017-06 \\
\hline Doc URL & http://hdl.handle.net/2115/70627 \\
\hline Rights & ○ 2017 The Japan Society of A pplied Physics \\
\hline Type & article (author version) \\
\hline File Information & BN170016.pdf \\
\hline
\end{tabular}

Instructions for use 


\title{
Excitation of cavitation bubbles in low-temperature liquid nitrogen
}

\author{
Koichi Sasaki* and Shingo Harada
}

Division of Quantum Science and Engineering, Hokkaido University, Sapporo 060-8628, Japan

\begin{abstract}
We excited a cavitation bubble by irradiating a Nd:YAG laser pulse onto a titanium target that was installed in liquid nitrogen at a temperature below the boiling point. To our knowledge, this is the first experiment in which a cavitation bubble has been successfully excited in liquid nitrogen. We compared the cavitation bubble in liquid nitrogen with that in water on the basis of an equation reported by Florschuetz and Chao [J. Heat Transfer 87, 209 (1965)].
\end{abstract}

Liquid-phase laser ablation attracts considerable attention as a new method for synthesizing functional nanomaterials. ${ }^{1,2)}$ This method has an advantage in the use of no chemicals. Crystalline nanoparticles with unique structures are synthesized, ${ }^{3-7)}$ which is another advantage of liquid-phase laser ablation. A mechanism for the synthesis of crystalline nanoparticles is considered to be related to the high pressure of the plasma produced by laser irradiation in a liquid environment. ${ }^{8)}$ In addition, the role of a cavitation bubble in the synthesis of nanoparticles has been pointed out recently. It has been shown that the reaction field for the growth of nanoparticles is the inside of the cavitation bubble. ${ }^{9-12)}$ Since the cavitation bubble at its collapse has a high pressure, ${ }^{13)}$ the structure of synthesized nanoparticles is affected by the dynamics of the cavitation bubble. ${ }^{14,15)}$

The medium used most widely in liquid-phase laser ablation is water. Nanoparticles of noble metals and oxidized materials are synthesized by laser ablation in water. On the other hand, approximately ten years ago, we tried laser ablation in liquid nitrogen as a method for synthesizing nitride nanoparticles. ${ }^{16)}$ However, the cavitation bubble was not excited in liquid nitrogen at $77 \mathrm{~K}$ in atmospheric pressure. ${ }^{17,18)}$ Therefore, we could not utilize the high pressure of the collapsed cavitation bubble in the synthesis of nitride nanoparticles by laser ablation in liquid nitrogen. In this work, we demonstrated the excitation of cavitation bubbles in liquid nitrogen by reducing its temperature below the boiling point. To our knowledge, this is the first experiment in which cavitation bubbles have been excited in liquid nitrogen.

The experimental apparatus used is schematically shown in Fig. 1. We prepared a coaxial

${ }^{*}$ E-mail address: sasaki@qe.eng.hokudai.ac.jp 
cylindrical chamber composed of four shells to realize laser ablation in liquid nitrogen at various temperatures. The four shells had quartz windows. The outermost shell (shell 1 in Fig. 1) was evacuated using a turbomolecular pump for thermal isolation. The second shell (shell 2) was filled with liquid nitrogen. Although liquid nitrogen in shell 2 was always at the boiling point, it was possible to control the temperature below $77 \mathrm{~K}$ by reducing its pressure using an oil rotary pump. The third shell (shell 3) was also filled with liquid nitrogen. The pressure in shell 3 was controlled between 0.1 and $0.2 \mathrm{MPa}$. The liquid nitrogen in shell 3 was cooled down by thermal conduction from shell 2 to obtain liquid nitrogen at a temperature lower than the boiling point. The innermost shell (shell 4) was a small cylinder for collecting nanoparticles after the experiment. Liquid nitrogen in shell 4 had the same temperature and the same pressure as those in shell 3.

A titanium target was installed on a rotatable holder in shell 4. We used a Nd:YAG laser at a wavelength of $532 \mathrm{~nm}$ to ablate the titanium target. The energy and duration of the Nd:YAG laser pulse were $60 \mathrm{~mJ}$ and $8 \mathrm{~ns}$, respectively. A lens was placed in shell 2 to focus the laser beam onto the target. The region in front of the target was observed by shadowgraph imaging. The light source was a metal halide lamp, and the pattern of the transmitted lamp light was captured using a high-speed camera with a flame rate of $200 \mathrm{kHz}$. The high-speed camera was replaced by a charge-coupled device camera with a gated image intensifier (ICCD camera) when we needed a higher temporal resolution. The temperature of liquid nitrogen was monitored by measuring the electric resistance of a platinum wire.

Figure 2 shows the shadowgraph images observed when the pressure and temperature of liquid nitrogen in shell 4 were $0.1 \mathrm{MPa}$ and $77 \mathrm{~K}$, respectively. The images were extracted from the high-speed movie. The times indicated below the images are the delay times after the irradiation of the Nd:YAG laser pulse. As shown in Figs. 2(a)-2(c), we observed the formation and expansion of a bubble from the irradiation point of the Nd:YAG laser pulse. After the maximum size was observed at $\sim 170 \mu$ s after the laser irradiation, we observed the change in bubble shape from hemispherical to distorted, as shown in Figs. 2(d)-2(f). After that, we observed that the bubble was shredded into small bubbles, as shown in Figs. $2(\mathrm{~g})$ 2(i). The shrinkage of the bubble was never observed when the pressure and temperature were $0.1 \mathrm{MPa}$ and $77 \mathrm{~K}$, respectively. In contrast, we observed the shrinkage of the bubble when we cooled the liquid nitrogen below the boiling point. Figure 3 shows the shadowgraph images observed when the pressure and temperature of liquid nitrogen in shell 4 were 0.1 $\mathrm{MPa}$ and $66 \mathrm{~K}$, respectively. After the maximum size was observed at $\sim 155 \mu$ s after the laser irradiation [Fig. 3(c)], we observed the shrinkage of the bubble as shown in Figs. 3(d)- 
3(f). The shrinkage was followed by the collapse and reformation of the secondary bubble, as shown in Figs. 3(g)-3(i). The maximum size of the secondary bubble was smaller than that of the first bubble.

The temporal variations of the bubble sizes, which were observed under three experimental conditions, are plotted in Fig. 4. Although the shape of the bubble induced at a temperature of $77 \mathrm{~K}$ and a pressure of $0.1 \mathrm{MPa}$ was not hemispherical after the maximum size, the distance between the target surface and the leading edge of the bubble is plotted in Fig. 4. The shrinkage, collapse, and rebound of the bubble were clearly observed when the temperature of liquid nitrogen was $66 \mathrm{~K}$. Note here that the secondary bubble had a similar lifetime to the first bubble in spite of its smaller size when the pressure was 0.1 MPa. When we pressurized the liquid nitrogen in shell 4 up to $0.2 \mathrm{MPa}$, we observed a smaller bubble with a shorter lifetime, as shown in Fig. 3. In this case, we observed the formation of the third cavitation bubble after the collapse of the second bubble.

The bubble radii plotted in Fig. 4 were obtained from movies captured using a high-speed camera. Since the temporal resolution of the high-speed camera $(5 \mu \mathrm{s})$ was insufficient to capture the collapsed bubble, we examined the the size of the bubble at its collapse using an ICCD camera with a gate width of $50 \mathrm{~ns}$. As a result, the radius of the collapsed cavitation bubble, which was induced at a pressure of $0.2 \mathrm{MPa}$ and a temperature of $66 \mathrm{~K}$, was identified to be $0.16 \mathrm{~mm}$. A shockwave was not generated at the collapse of the bubble, even though the gate width of $50 \mathrm{~ns}$ was sufficient to capture the wavefront of the shockwave with a propagation speed of approximately $1100 \mathrm{~m} / \mathrm{s}$.

Florschuetz and Chao have reported that the bubble dynamics is understood on the basis of the parameter defined by ${ }^{19)}$

$$
B_{\text {eff }}=\psi^{2}\left[\frac{\rho c\left(T_{\mathrm{b}}-T_{\infty}\right)}{\bar{\rho}_{\mathrm{v}} L}\right]^{2} \frac{\kappa}{R_{0}}\left(\frac{\rho}{p_{\infty}-p_{\mathrm{v} 0}}\right)^{1 / 2},
$$

where $\rho, c, \kappa$, and $T_{\mathrm{b}}$ are the density, specific heat, thermal diffusivity, and boiling point of the liquid, respectively, $L$ is the latent heat, $R_{0}$ is the maximum bubble radius, $T_{\infty}$ is the temperature of the liquid at a long distance from the bubble, and $p_{\mathrm{v} 0}$ and $p_{\infty}$ are the vapor pressures at $T_{\infty}$ and $T_{\mathrm{b}}$, respectively. $\psi$ is called the temperature difference correction factor, which is given by

$$
\psi=\frac{2}{\left(T_{\mathrm{b}}-T_{\infty}\right)\left(p_{\infty}-p_{\mathrm{v} 0}\right)} \int_{T_{\infty}}^{T_{\mathrm{b}}}\left[p_{\infty}-p_{\mathrm{v}}(T)\right] \mathrm{d} T,
$$

where $p_{\mathrm{v}}(T)$ represents the temperature dependence of the vapor pressure, and $\bar{\rho}_{\mathrm{v}}$ is the aver- 
age vapor density defined by

$$
\bar{\rho}_{\mathrm{v}}=\frac{1}{T_{\mathrm{b}}-T_{\infty}} \int_{T_{\infty}}^{T_{\mathrm{b}}} \rho_{\mathrm{v}}(T) \mathrm{d} T,
$$

where $\rho_{\mathrm{v}}(T)$ represents the temperature dependence of the equilibrium vapor density. According to Florschuetz and Chao, the bubble dynamics is dominated by the liquid inertia when $B_{\text {eff }} \geq 10$, and in this case, the hard collapse of the bubble is realized. On the other hand, when $B_{\text {eff }} \leq 0.05$, the heat transfer controls the bubble dynamics, and in this case, the shrinkage and collapse of the bubble become weaker.

Table I shows the $B_{\text {eff }}$ values obtained under the experimental conditions in Fig. 4 . $B_{\text {eff }}$ under a typical experimental condition of laser ablation in water is also shown in Table I for comparison. We ignored the temperature dependences of the thermodynamic parameters in the evaluation of $B_{\text {eff }}$. As shown in Table I, $B_{\text {eff }} \geq 10$ is realized in water, such that we observe the hard collapse of the cavitation bubble with the generation of a shockwave in laser ablation in water. ${ }^{20)}$ The experimental result shown in Fig. 2 is understood reasonably by considering $B_{\text {eff }}=0$ in liquid nitrogen at $77 \mathrm{~K}$ (boiling point).

$B_{\text {eff }}$ in liquid nitrogen is much smaller than that in water even when the temperature was reduced below the boiling point. The reasons for the small $B_{\text {eff }}$ in liquid nitrogen are the small temperature difference from the boiling point and the large equilibrium vapor density. The maximum $B_{\text {eff }}$ we realized in this experiment was $1.2 \times 10^{-2}$. The heat transfer controls the bubble dynamics under the present experimental conditions according to Florschuetz and Chao, but we realized the bubbles with the dynamics of shrinkage, collapse, and rebound. Because of these dynamics, we may categorize the bubble as "a cavitation bubble". However, the dynamics of the cavitation bubbles in liquid nitrogen was less energetic than that in water. The less energetic dynamics is understood by the large radius of the collapsed bubble $(0.16$ $\mathrm{mm}$ in liquid nitrogen and $0.07 \mathrm{~mm}$ in $w^{2} \mathrm{ter}^{20)}$ ), the negligible generation of the shockwave, and the long lifetime of the secondary bubble at a temperature of $66 \mathrm{~K}$ and a pressure of 0.1 MPa.

In conclusion, we have succeeded in the excitation of a cavitation bubble with the dynamics of shrinkage, collapse, and rebound in liquid nitrogen by reducing its temperature below the boiling point. To our knowledge, this is the first experiment in which a cavitation bubble has been successfully excited in liquid nitrogen. However, the dynamics of the cavitation bubble in liquid nitrogen was less energetic than that in water, which is understood by fact that $B_{\text {eff }}$ in liquid nitrogen is much smaller than that in water. 


\section{References}

1) F. Mafuné, J. Kohno, Y. Takeda, T. Kondow, and H. Sawabe, J. Phys. Chem. B 104, 8333 (2000).

2) F. Mafuné, J. Kohno, Y. Takeda, T. Kondow, and H. Sawabe, J. Phys. Chem. B 104, 9111 (2000).

3) G. W. Yang, J. B. Wang, and Q. X. Liu, J. Phys.: Condens. Matter 10, 7923 (1998).

4) P. Liu, Y. L. Cao, H. Cui, X. Y. Chen, and G. W. Yang, Chem. Mater. 20, 494 (2008).

5) X. Y. Chen, H. Cui, P. Liu, and G. W. Yang, Chem. Mater. 20, 2035 (2008).

6) C. H. Liang, Y. Shimizu, T. Sasaki, and N. Koshizaki, Appl. Phys. A 80, 819 (2005).

7) D. Amans, A.-C. Chenus, G. Ledoux, C. Dujardin, C. Reynaud, O. Sublemontier, K. Masenelli-Varlot, and O. Guillois, Diamond Relat. Mater. 18, 177 (2009).

8) H. Ushida, N. Takada, and K. Sasaki, J. Phys.: Conf. Ser. 59, 563 (2007).

9) W. Soliman, N. Takada, and K. Sasaki, Appl. Phys. Express 3, 035201 (2010).

10) S. Ibrahimkutty, P. Wagener, A. Menzel, A. Plech, and S. Barcikowski, Appl. Phys. Lett. 101, 103014 (2012).

11) S. Ibrahimkutty, P. Wagener, T. S. Rolo, D. Karpov, A. Menzel, T. Baumbach, S. Barcikowski, and A. Plech, Sci. Rep. 5, 16313 (2015).

12) M. Takeuchi and K. Sasaki, Appl. Phys. A 122, 312 (2016).

13) C. Brennen, Cavitation and Bubble Dynamics (Oxford University Press, Oxford, U.K., 1995).

14) W. Soliman, N. Takada, N. Koshizaki, and K. Sasaki, Appl. Phys. A 110, 779 (2013).

15) N. Takada, Fujikawa, N. Koshizaki, and K. Sasaki, Appl. Phys. A 110, 835 (2013).

16) N. Takada, T. Sasaki, and K. Sasaki, Appl. Phys. A 93, 833 (2008).

17) N. Takada, T. Nakano, and K. Sasaki, Appl. Phys. A 101, 255 (2010).

18) H. Sato, X. W. Sun, M. Odagawa, K. Maeno, and H. Honma, J. Fluids Eng. 118, 850 (1996).

19) L. W. Florschuetz and B. T. Chao, J. Heat Transfer 87, 209 (1965).

20) K. Sasaki, T. Nakano, W. Soliman, and N. Takada, Appl. Phys. Express 2, 046501 (2009). 


\section{Figure captions}

Fig. 1. (Color online) Schematic of the experimental apparatus.

Fig. 2. Shadowgraph images observed at various delay times after the irradiation of the Nd:YAG laser pulse: (a) $0 \mu \mathrm{s}$, (b) $5 \mu \mathrm{s}$, (c) $125 \mu \mathrm{s}$, (d) $325 \mu \mathrm{s}$, (e) $400 \mu \mathrm{s}$, (f) $3.8 \mathrm{~ms}$, (g) $5.7 \mathrm{~ms}$, (h) $13 \mathrm{~ms}$, and (i) $19 \mathrm{~ms}$. The pressure and temperature of liquid nitrogen were $0.1 \mathrm{MPa}$ and $77 \mathrm{~K}$, respectively.

Fig. 3. Shadowgraph images observed at various delay times after the irradiation of the Nd:YAG laser pulse: (a) $0 \mu \mathrm{s}$, (b) $45 \mu \mathrm{s}$, (c) $155 \mu \mathrm{s}$, (d) $250 \mu \mathrm{s}$, (e) $275 \mu \mathrm{s}$, (f) $300 \mu \mathrm{s},(\mathrm{g}$ ) $440 \mu \mathrm{s}$, (h) $580 \mu \mathrm{s}$, and (i) $640 \mu \mathrm{s}$. The pressure and temperature of liquid nitrogen were $0.1 \mathrm{MPa}$ and $66 \mathrm{~K}$, respectively.

Fig. 4. (Color online) Temporal variation in bubble radius after the irradiation of the Nd:YAG laser pulse. 
Table I. Evaluation of $B_{\text {eff }}$ given by Eq. (1) under the present experimental conditions. $B_{\text {eff }}$ under a typical condition of laser ablation in water is also shown for comparison.

\begin{tabular}{ccccccc}
\hline \hline Medium & $p_{\infty}(\mathrm{MPa})$ & $T_{\infty}(\mathrm{K})$ & $T_{\mathrm{b}}-T_{\infty}(\mathrm{K})$ & $\bar{\rho}_{\mathrm{v}}\left(\mathrm{kg} / \mathrm{m}^{3}\right)$ & $R_{0}(\mathrm{~mm})$ & $B_{\text {eff }}$ \\
\hline Liquid $_{2}$ & 0.1 & 77 & 0 & 4.4 & 1.5 & 0 \\
Liquid $_{2}$ & 0.1 & 66 & 11 & 2.4 & 1.8 & $0.9 \times 10^{-2}$ \\
Liquid $\mathrm{N}_{2}$ & 0.2 & 66 & 18 & 3.6 & 1.3 & $1.2 \times 10^{-2}$ \\
Water & 0.1 & 300 & 73 & 0.2 & 1 & 13 \\
\hline \hline
\end{tabular}




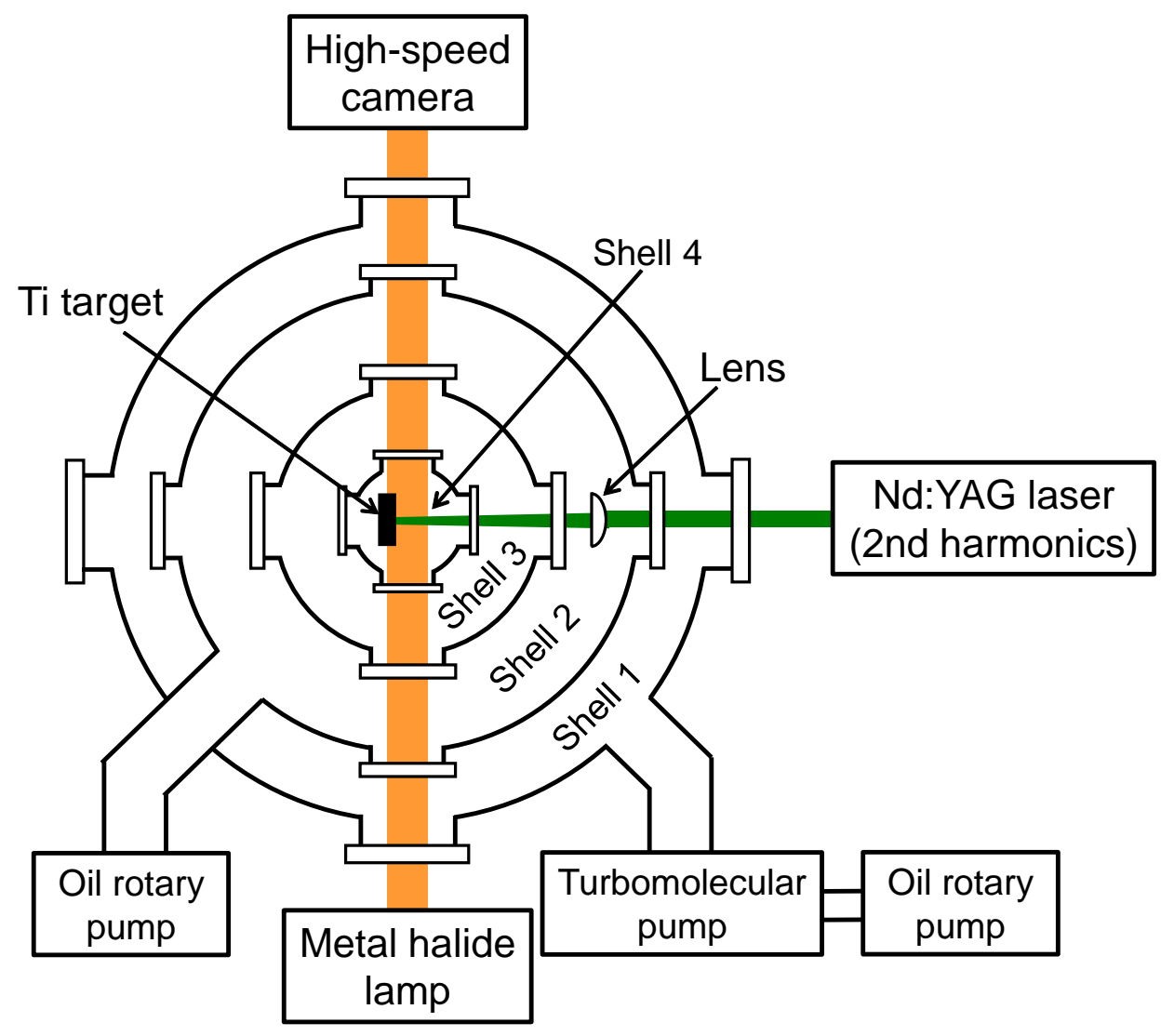

Fig. 1. (Color online) Schematic of the experimental apparatus. 


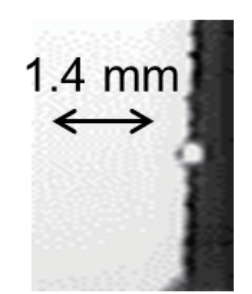

(a)

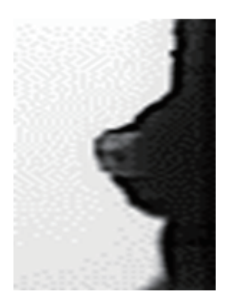

(d)

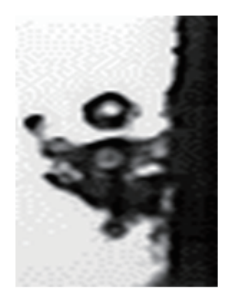

(g)

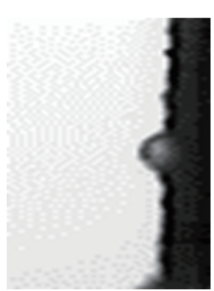

(b)

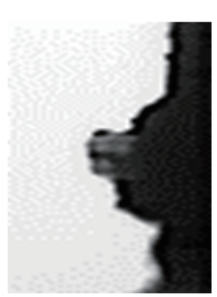

(e)

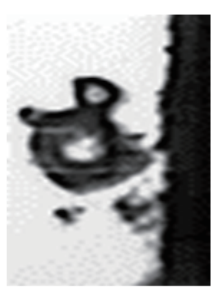

(h)

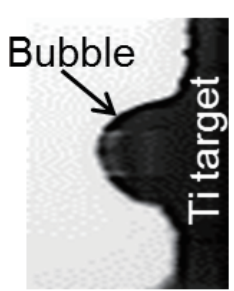

(c)

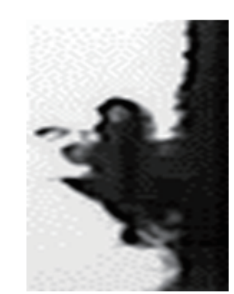

(f)

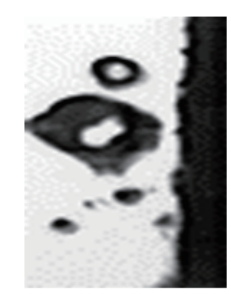

(i)

Fig. 2. Shadowgraph images observed at various delay times after the irradiation of the Nd:YAG laser pulse: (a) $0 \mu \mathrm{s}$, (b) $5 \mu \mathrm{s}$, (c) $125 \mu \mathrm{s}$, (d) $325 \mu \mathrm{s}$, (e) $400 \mu \mathrm{s}$, (f) $3.8 \mathrm{~ms}$, (g) $5.7 \mathrm{~ms}$, (h) $13 \mathrm{~ms}$, and (i) $19 \mathrm{~ms}$. The pressure and temperature of liquid nitrogen were $0.1 \mathrm{MPa}$ and $77 \mathrm{~K}$, respectively. 


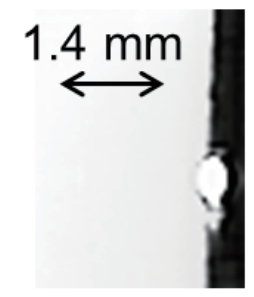

(a)

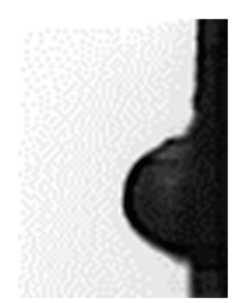

(d)

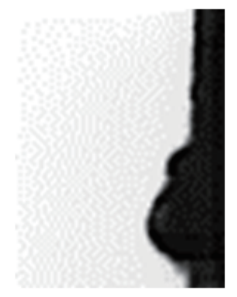

(g)

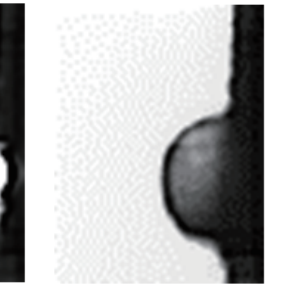

(b)

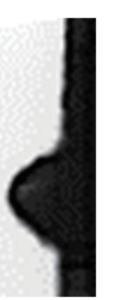

(e)

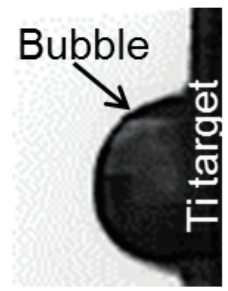

(c)

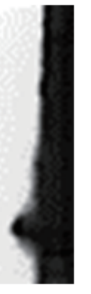

(h)

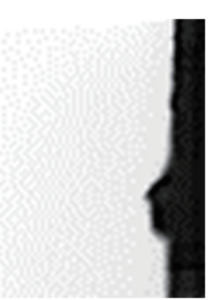

(f)

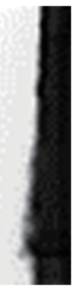

(i)

Fig. 3. Shadowgraph images observed at various delay times after the irradiation of the Nd:YAG laser pulse: (a) $0 \mu \mathrm{s}$, (b) $45 \mu \mathrm{s}$, (c) $155 \mu \mathrm{s}$, (d) $250 \mu \mathrm{s}$, (e) $275 \mu \mathrm{s}$, (f) $300 \mu \mathrm{s}$, (g) $440 \mu \mathrm{s}$, (h) $580 \mu \mathrm{s}$, and (i) $640 \mu \mathrm{s}$. The pressure and temperature of liquid nitrogen were $0.1 \mathrm{MPa}$ and $66 \mathrm{~K}$, respectively. 


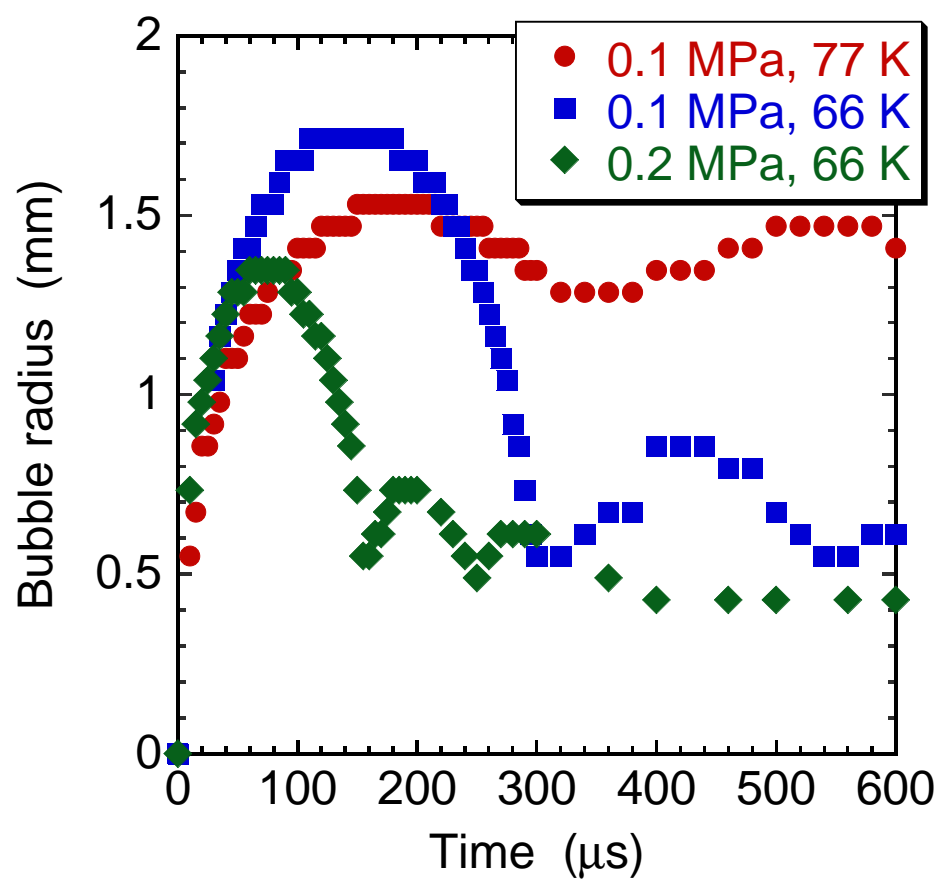

Fig. 4. (Color online) Temporal variation in bubble radius after the irradiation of the Nd:YAG laser pulse. 